Denis Duboule Paolo Sordino

\section{Des nageoires aux membres : l'apport de la génétique moléculaire du développement dans l'étude de l'évolution des morphologies chez les vertébrés}

Les cinq dernières années ont vu la découverte de plusieurs gènes impliqués dans la morphogenèse des membres des tétrapodes, permettant une nouvelle approche des mécanismes responsables de l'ontogenèse de ces structures complexes. En particulier, ces gènes peuvent servir d'outils pour réexaminer quelques aspects d'une des questions les plus discutées de ce siècle en paléontologie des vertébrés : le passage d'une nageoire ancestrale à des membres authentiques de tétrapodes, c'est-à-dire munis de ces éléments fascinants et indispensables que sont les doigts. Les comparaisons moléculaires entre poissons et mammifères renforcent le lien phylogénétique entre ces structures, pourtant d'apparence si différente, mais révèlent également des différences fondamentales. En particulier, l'étude des gènes $H o x$ d'un petit poisson téléostéen (le poisson-zèbre, Danio rerio) suggère un schéma de transition nageoire-membre dans lequel les extrémités des mains et des pieds (les doigts) sont des structures néomorphes produites par prolifération inégale de la partie postérieure et distale d'une nageoire-membre ancestrale.

D. Duboule: professeur à la faculté des sciences de l'université de Genève, directeur du laboratoire d'embryologie moléculaire et morphogenèse. P. Sordino: biologiste, assistant à la faculté des sciences de l'université de Genève. Département de zoologie et biologie animale, université de Genève, Sciences III, Quai Ernest-Ansermet 30, 1211 Genève 4, Suisse.

'origine et la transformation des appendices latéraux représentent un trait fondamental de l'évolution des vertébrés. Depuis la définition des homologies de structure entre les membres de tous les tétrapodes
[1], on a proposé l'existence d'un plan de développement unique [2] suivant lequel des instructions de base constitueraient un contexte d'informations permettant l'élaboration de spécialisations néo-ontologiques pour chaque espèce [3]. Sur 


\section{RÉFÉRENCES}

1. Owen R. On the nature of limbs. London : Van der Hoorst J, 1849.

2. Gegenbaur C. Ueber das archipterygium. Jena $Z$ Naturw 1872; 7: 131-41.

3. Duboule D. How to make a limb? Science 1994 ; 266: 575-6.

4. Coates MI. The origin of vertebrate limbs. Development 1994; suppl: 169-80.

5. Shubin N. The evolution of paired fins and the origin of tetrapod limbs. Phylogenetic and transformational approaches. In : Hecht MK, Macintyre RJ, Clegg MT, eds. Evolutionary biology. New York: Plenum Press, 1995: 39-86.

6. Slack JMW, Holland PWH, Graham CF. The zootype and the phylotypic stage. $\mathrm{Na}$ ture $1993 ; 361: 490-2$.

7. Duboule D. Temporal colinearity and the phylotypic progression: a basis for the stability of a vertebrate Bauplan and the evolution of morphologies through heterochrony. Development 1994; suppl : 135-42.

8. Hinchliffe JR, Johnson DR. The develop ment of the vertebrate limb. Oxford: Oxford University Press, 1980.

9. Coates MI. Hox genes, fin folds and symmetry. Nature 1993 ; 364: 195-6.

10. Jarvik E. Basic structure and evolution in vertebrates. New York: Academic Press, 1980.

11. Coates MI, Clack JA. Polydactyly in the earliest known tetrapod limbs. Nature 1990 ; $347: 66-9$

12. Vorobyeva EI, Schultze HP. Description and systematics of panderichthyd fishes with comments on their relationship to te trapods. In: Schultze HP, Trueb L, eds. Ongin of the higher groups of tetrapods. Ithaca: Comstock Publishing Associates, 1991: 68-

la base de données paléontologiques et embryologiques (expérimentales et comparatives), plusieurs tentatives ont été faites pour présenter une synthèse globale de ce problème fascinant [4, 5]. Pourtant, nos connaissances sont encore réduites, tant en ce qui concerne la forme ancestrale de nos membres que la séquence de transformations morphologiques entre les vertébrés inférieurs et supérieurs, et sont, malheureusement, loin de permettre une définition claire de l'histoire évolutive de nos bras et jambes. Dans ce contexte, l'étude comparative des mécanismes moléculaires de la morphogenèse des membres peut se révéler fructueuse. Le fait que les systèmes biomoléculaires impliqués ont retenu d'importantes similitudes pendant des millions d'années (mia) suggère que des variations minimales, à l'intérieur de ces systèmes, ont été de riches sources d'évolution morphologique (macroévolution) [6, 7]. Apprendre comment des molécules régulatrices, conservées au plan phylogénétique, ont pu engendrer des variations de morphologie requiert des comparaisons fonctionnelles détaillées dans une perspective évolutive. Dans cette revue, nous aimerions à la fois discuter quelques résultats récents, obtenus en étudiant la morphogenèse des nageoires des téléostéens, et tenter d'évaluer à quel point des scénarios évolutifs construits à partir de données paléontologiques et d'observations ontogénétiques peuvent être corroborés par une analyse comparative de comportements moléculaires.

Il est généralement admis que certains chordés primitifs avaient un bourrelet médian continu, s'étendant de la partie dorsale jusqu'à la face ventrale en passant par la queue. Ce repli, voisin de celui existant chez les céphalocordés et certaines larves d'urocordés, aurait permis des mouvements ondulatoires dans un milieu aquatique. Il est possible qu'un repli similaire, mais s'étendant latéralement de part et d'autre de l'animal, soit apparu, repli qui pourrait donc représenter la première ébauche de structures latérales lors de l'évolution des vertébrés primitifs. Les ostracodermes (vertébrés sans mâchoires de l'Ordovicien et du Dévonien; 480-350 mia) avaient des structures quelque peu différentes [4], antérieures à des nageoires authentiques, dont la fonction était probablement plus liée à la stabilité qu'à la propulsion. La première manifestation d'une vraie paire de nageoires flexibles (cousines de celles des poissons modernes) remonte à la fin du Silurien (370 mia) [4, 8] et fut trouvée chez des fossiles de poissons à mâchoires. Là encore, hélas, l'origine mystérieuse de ces appendices ne permet pas de statuer sur la nature des structures ancestrales à partir desquelles de tels squelettes appendiculaires se sont développés [9]. Ainsi, l'histoire de l'apparition des nageoires reste matière à spéculation. Mais il est certain qu'une telle innovation morphologique représenta une solution avantageuse au problème de la locomotion, comme en témoigne l'étonnant éventail d'adaptations réussies de ces structures à leurs milieux. De plus, l'émergence et la stabilisation de la pentadactylie, parallèlement à l'augmentation de la taille du cerveau, ont probablement été des facteurs importants dans l'acquisition de fonctions complexes chez les primates [10].

L'analyse de fossiles suggère que la transition phénotypique de la nageoire au membre se déroula en parallèle à la transformation des poissons à nageoires lobées en vertébrés terrestres (Dévonien; 350$370 \mathrm{mia})$. Cette transition s'accomplit en parallèle à une utilisation toujours plus importante du sol, à l'origine dans des habitats peu profonds comme en témoigne la nature aquatique des tétrapodes les plus anciens, tels que Ichthyostega et Acanthostega [11]. Des poissons du Dévonien, de la famille des Panderichthyidae [12] pourraient ainsi représenter les parents les plus proches d'un tétrapode ancestral [13]. En effet, l'endosquelette de leurs nageoires pectorales montre d'importantes similitudes avec celui de membres authentiques, attestées par la présence d'un humérus prolongé par deux os, le radius et l'ulna (figure 1). C'est 


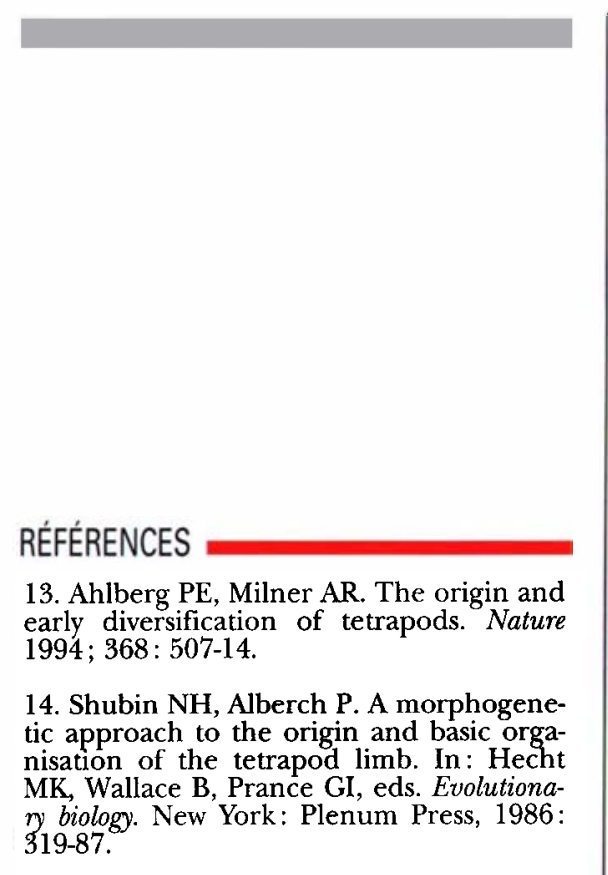

15. Saunders JW, Gasseling MT. Ectodermal-mesenchymal interactions in the origin of limb symmetry. In : Fleischmajer $\mathbf{R}$ gillingham RE, eds. Epithelial-mesenchymal interactions. Baltimore: Williams and Wilkins, 1968: 78-97.

16. Concordet J. Morphogenèse, acide rétinoïque et Sonic Hedgehog. médecine/sciences 1994; 10:570-3.

17. Geraudie $J$. The fine structure of the early pelvic fin bud of the trouts Salmo gairdneri and S. trutta fario. Acta Zool 1978; 59: 85-96.

18. Wood A. Early pectoral fin development and morphogenesis of the apical ectodermal ridge in the killifish Aphysosemion scheeli. Anat Rec 1982; 204: 349-56.

19. Thorogood P. The development of the teleost fin and implications for our understanding of tetrapod limb evolution. In: Hinchliffe JR, Hurle JM, Summerbell D, eds. Developmental patterning of the vertebrate limb. New York: Plenum Press, 1991 : 34754.

20. Concordet JP, Ingham P. Catch of the decade. Nature 1994; 369 : 19-20.

21. Sordino P, van der Hoeven F, Duboule D. Hox gene expression in teleost fins and the origin of vertebrate digits. Nature 1995 $375: 678-81$.

22. Van der Hoeven F, Sordino P, Fraudeau N, Izpisua-Belmonte JC, Duboule D. Teleost $H o x D$ and HoxA genes: comparison with tetrapods and functional evolution of the HoxD complex. Development 1996 (sous presse).

23. Dollé $P$, Izpisua-Belmonte JC, Falkenstein H, Renucci A, Duboule D. Coordinate expression of the murine Hox-5 complex homeobox-containing genes during limb pattern formation. Nature $1989 ; 342$ : 767 pat.

$\mathrm{m} / \mathrm{s} n^{\circ} 2$, vol. 12, férier 96

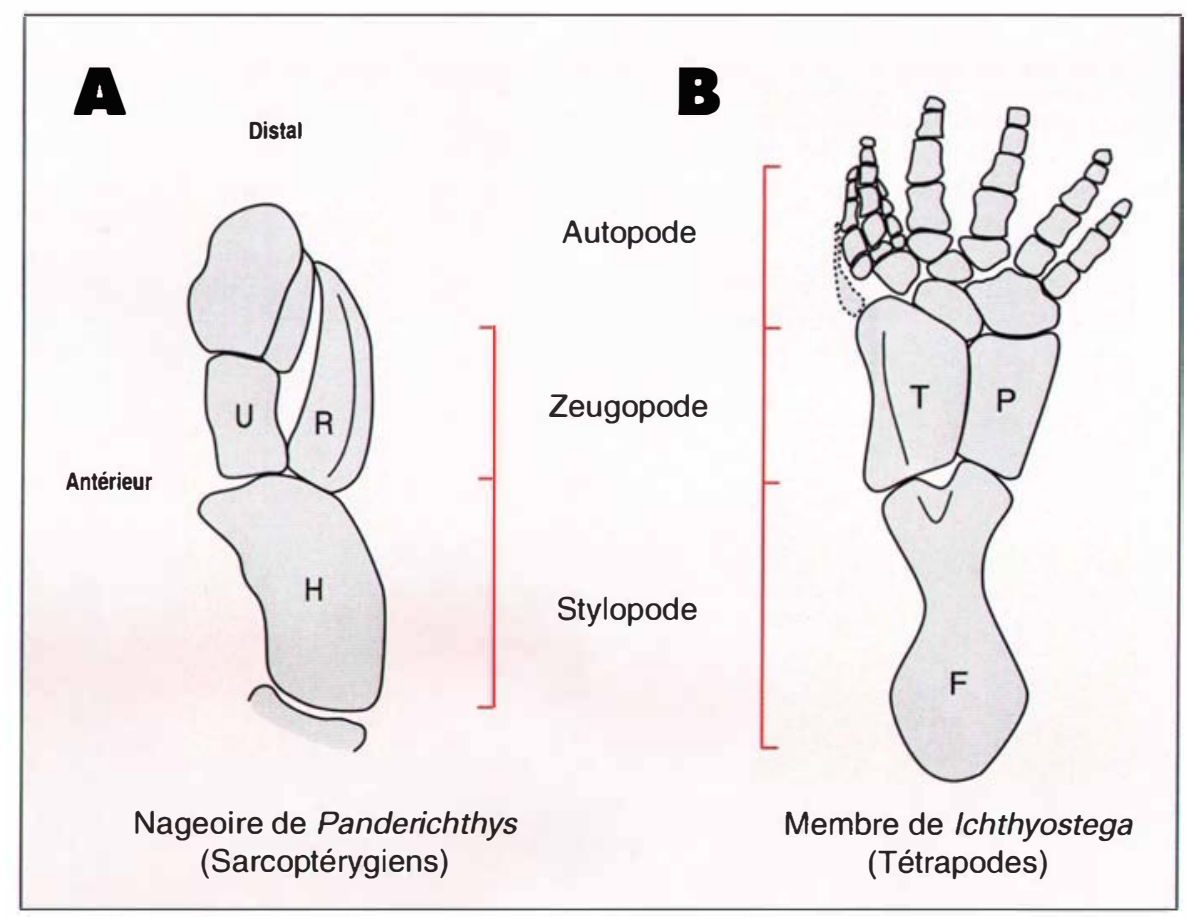

Figure 1. Deux étapes possibles dans la transition de la nageoire au membre, illustrées par des fossiles du Dévonien. A gauche, une nageoire de Panderichthis, comprenant à la fois un stylopode (bras) et un zeugopode (avant-bras); à droite, le membre d'un tétrapode ancestral, Ichthyostega, avec l'apparition $d^{\prime}$ 'un autopode (main, pied). Les éléments osseux homologues sont indiqués: $H$, humérus; $U$, ulna (cubitus); $R$, radius; $F$, fémur; $P$, péroné; $T$, tibia.

sur la base de cet élément de structure que les nageoires pectorales et pelviennes sont définies comme homologues des membres antérieurs et postérieurs des vertébrés supérieurs. Cependant, tant chez Panderichtys que chez les poissons à nageoires lobées, aucun élément osseux ne peut être clairement considéré comme homologue des extrémités distales des tétrapodes.

La grande majorité des membres des tétrapodes se développent et s'organisent d'une façon identique ([14] et figure 2). Initialement, des cellules mésenchymateuses des plaques latérales bourgeonnent et induisent la différenciation d'une couche ectodermique spécialisée: la crête apicale ectodermique (CAE). Grâce à la sécrétion de différents facteurs, cette couche d'ectoderme maintient à son tour le mésoderme sous-jacent dans un état prolifératif. Dans cette zone (la zone de progrès, $\mathrm{PZ}$ ), les cellules ne se condensent pas. La face postérieure du bourgeon de membre contient une activité «organisatrice» (ZPA), mise en évidence par sa capacité d'induire une duplication en miroir de la digitation quand elle est greffée à la marge antérieure d'un bourgeon receveur $[15,16]$. Le squelette du membre est donc établi d'une façon progressive, comme une série de condensations préchondrogéniques, dans lesquelles sont impliquées les cellules qui ne se trouvent plus sous l'influence de la couche ectodermique. Il en découle que la morphogenèse du membre suit une progression proximo-distale, débutant par la production du stylopode (le bras), suivie par le zeugopode (l'avant-bras) pour se terminer par l'autopode (la main).

Chez les poissons, tant cartilagineux qu'osseux, le développement des nageoires paires semble être contrôlé par des mécanismes voisins (figure 2). Un épaississement initial du mésoderme latéral est suivi par l'émergence d'une couche ectodermique un peu particulière, une pseudo-CAE [17, 18], ou repli ectodermique apical (REA). Peu après le bourgeonnement, cette couche se replie sur elle-même et s'allonge pour servir de gaine au futur squelette dermique de la nageoire (figu- 


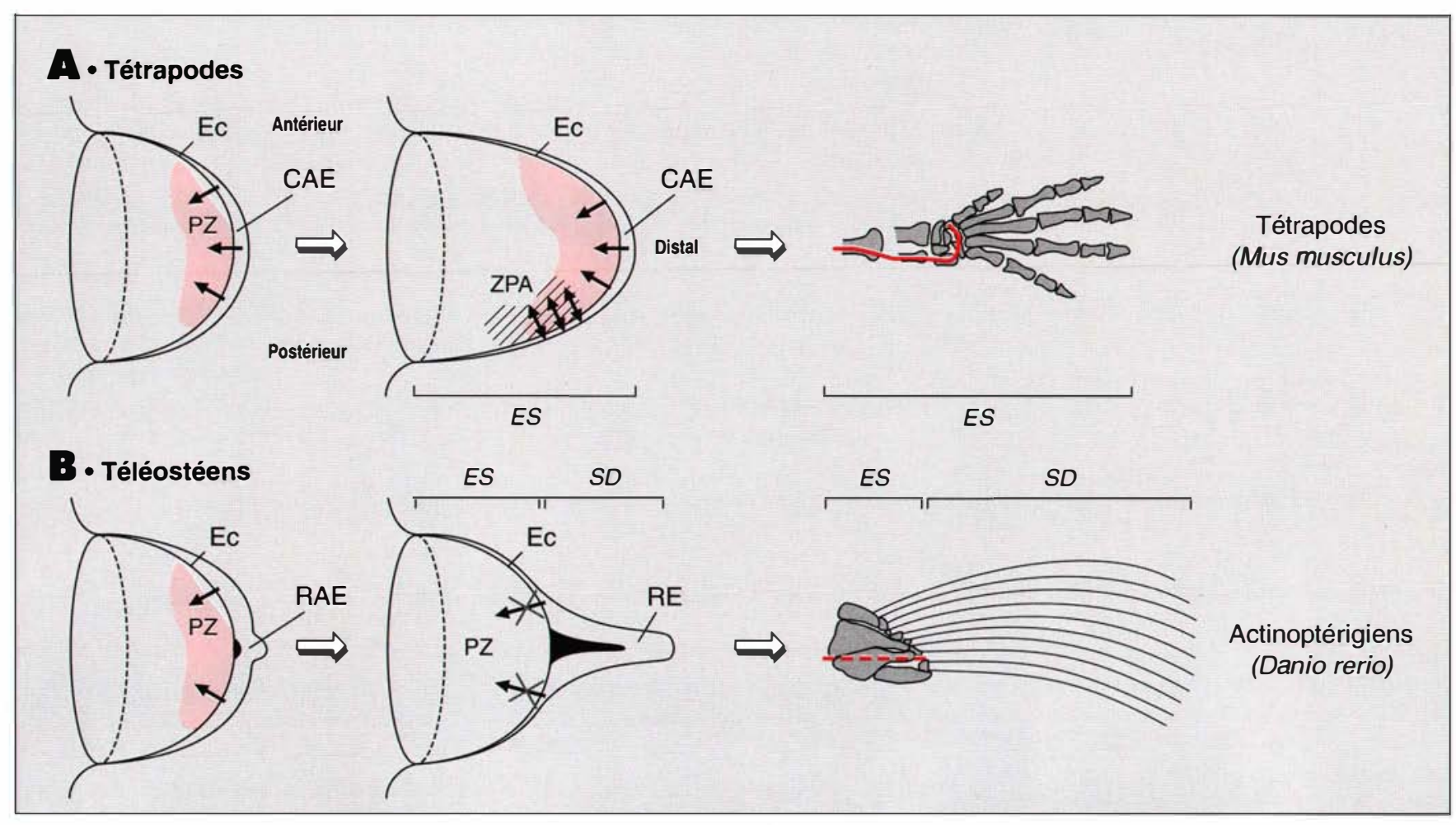

Figure 2. Description schématique du développement des appendices latéraux chez les tétrapodes (A) et les poissons téléostéens (B). Deux stades de développement sont montrés ainsi qu'un schéma de la morphologie finale. Ces stades correspondent aux deux phases d'expression des gènes Hox (voir aussi figure 4); d'abord, une phase correspondant à une croissance symétrique, observée pendant et après le bourgeonnement, contrôlée par la totalité de la couche ectodermique (flèches). Cette première phase est pratiquement identique chez la souris et le poisson. Ultérieurement, un second système génétique entre en jeu, impliquant une boucle rétroactive entre le mésenchyme et l'ectoderme, mais seulement dans la partie postérieure (flèches doubles). Cette seconde phase donne lieu à une sur-prolifération du mésenchyme postérieur qui va former l'autopode par un mécanisme de branchement post-axial (postérieur) alors que les autres éléments osseux (plus proximaux) sont produits par branchement préaxial (antérieur à l'axe majeur). L'axe majeur de l'appendice (ligne rouge épaisse) apparaîtra donc plié vers la marge antérieure, dans la structure adulte là droite). Chez le poisson cependant, cette phase secondaire n'est pas observée ainsi que l'indique la ligne rouge épaisse et discontinue. A la place, le repli de l'ectoderme, coïncidant avec l'apparition des actinotriches entre les deux couches (en noir), signe, peut être mécaniquement, l'interruption du contact ectoderme-mésenchyme et, par conséquent, de la prolifération du compartiment mésodermique. II en résultera un endosquelette réduit (en bas à droite). A droite, les squelettes des appendices adultes résultants sont dessinés, avec les proportions respectives de l'endosquelette (ES) comparé au squelette dermique (SD). Ec, ectoderme; $P Z$, zone de progrès; $C A E$, crête apicale ectodermique; $R A E$, repli apical ectodermique; $R E$, repli ectodermique de la nageoire; ES, endosquelette; $S D$, squelette dermique.

re $2 B, R A E)$. Le squelette des nageoires se construit donc à partir de deux composantes très différentes : d'une part, un endosquelette proximal, d'origine mésodermique, d'autre part, un squelette dermique plus ou moins flexible, dont l'origine embryologique n'est pas clairement définie. Le premier possède un nombre variable d'éléments, disposés en un ou plusieurs niveaux, qui sont homologues des os du squelette du membre des tétrapodes. Le second, qui fait la majeure partie de térisé par la flexibilité de ses rayons osseux (lépidotriches) et cartilagineux (actinotriches). Aucune structure homologue du squelette dermique des nageoires n'existe chez les tétrapodes.

Les poissons modernes, comme le Danio, ont un endosquelette réduit en comparaison de leur squelette dermique. Chez de tels spécimens, l'absence de structure homologue de l'autopode des tétrapodes est flagrante et résulte probablement d'un arrêt de la prolifération du futur endosquelette, simultanément au repli de la couche ectodermique décrit précédemment (figure 2). Il apparaît donc que la transition nageoire-membre impliqua la perte complète du squelette dermique et que l'étendue de l'endosquelette pourrait dépendre du moment auquel le repli ectodermique apparaît [19]. Chez les poissons à caractère plus "ancien", tels que le cœlacanthe (Latimeria chalumnae) et le poisson à poumon australien (Neoceratodus forsteri), un repli tardif permettrait une croissance supplémentaire de l'endosquelette, donc le 


\section{RÉFÉRENCES}

24. Dollé $P$, Dierich A, Schimmang $T$, Schuhbaur B, LeMeur M, Chambon P, Duboule $\mathrm{D}$. Disruption of the Hoxd-13 gene induces localized heterochrony leading to mice with neotenic limbs. Cell 1993; 75 . 431-41.

25. Davis AP, Witte DP, Hsieh-Li HM, Potter SS, Capecchi MR. Absence of radius and ulna in mice lacking Hoxa-11 and Hoxd-11. Nature 1995; $375: 791-5$.

26. Yokouchi Y, Sasaki H, Kuroiwa A. Homeobox gene expression correlated with the bifurcation process of limb cartilage development. Nature 1991 ; 353 : 443-45.

27. Haack H, Gruss $P$. The establishment of murine Hox-1 expression domains during patterning of the limb. Dev Biol 1993; 157: $410-29$

28. Gegenbaur C. Untersuchungen zur vergleichenden. In : Engelmann W, ed. Anatomie der wirbeltiere, band II. Leipzig : 1865.

29. Duboule D. Vertebrate Hox genes and proliferation : an alternative pathway to homeosis? Cur Opin Gen Dev 1995; 5: 525-8.

30. Niswander L, Jeffrey S, Martin GR, Tickle C. A positive feedback loop coordinates

growth and patterning in the vertebrate limb. Nature 1994; 371: 609-12.

31. Laufer E, Nelson CE, Johnson RL, Morgan BA, Tabin C. Sonic hedgehog and Fgf-4 act through a signaling cascade and feedback loop to integrate growth and patterning of the developing limb bud. Cell 1994 $79: 993-1004$

32. Orster GF, Shubin N, Murray JD, Alberch P. Evolution and morphogenetic rules: the shape of the vertebrate limb in ontogeny and phylogeny. Evolution 1988; 42 : 86284

33. Saunders JW. The experimental analysis of chick limb development. In : Ede DA, Hinchliffe JR, Balls M, eds. Vertebrate limb and somite morphogenesis. Cambridge: Cambridge University Press, 1977: 1-24.

34. Holmgreen N. An embryological analysis of the mammalian carpus and its bearing upon the question of the origin of the tetrapod limb. Acta Zool Stockh 1952; 33 : 1-115.

$\mathrm{m} / \mathrm{s} n^{\circ} 2$, vol. 12, féurier 96 développement d'une nageoire comportant davantage d'éléments osseux (voir plus loin). Par conséquent, la nageoire peut être considérée comme un membre réduit, tronqué dans la partie la plus distale de son endosquelette.

Dans le but d'évaluer à quel point les homologies fondées sur ces critères embryologiques et anatomiques sont confortées par des études de marqueurs moléculaires, nous avons isolé et caractérisé les gènes des complexes HoxA et HoxD chez les poissons téléostéens. Ces gènes sont d'une importance capitale dans le développement des membres des tétrapodes, chez lesquels ils contrôlent à la fois la présence et l'organisation des différentes structures. Le poisson zèbre (Danio rerio), un petit poisson tropical d'eau fraîche, se prête particulièrement bien aux études embryologiques et génétiques [20]. Cependant, ce poisson moderne possède des nageoires largement apomorphes, dans lesquelles l'endosquelette est très réduit à l'avantage d'un squelette dermique flexible bien développé. Il est donc évident que Danio ne possède pas une forme ancestrale de nageoire, mais au contraire une variante évoluée d'appendices latéraux bien adaptés au milieu aquatique.

Le clonage des gènes Hox chez Danio révéla que les vertébrés inférieurs possèdent déjà les quatre complexes de gènes que l'on retrouve chez les mammifères. Le passage des poissons aux tétrapodes n'a donc pas été accompagné d'une amplification du nombre de gènes Hox, ceux-ci étant déjà dans leur configuration la plus complète [21, 22]. L'expression de gènes appartenant aux complexes D et $\mathrm{A}$ a été analysée pendant le développement des nageoires pectorales et pelviennes [21]. Ces gènes, membres des groupes de paralogie 9 à 13, c'est-à-dire parents du gène $A b d o m i n a l B(A b d B)$ de la Drosophile, sont essentiels pour la morphogenèse des membres [23-27]. Leur inactivation fonctionnelle engendre des malformations des membres qui sont interprétées en termes de déficit, soit dans la prolifération cellulaire, soit dans les condensations préchondrogéniques [24, 25]. En particulier, les gènes $H o x D$ ont des profils d'expression complexes pendant le dévelop- pement des membres. Dans une première phase, ils sont exprimés selon une stratégie de poupées russes centrée sur la partie postérieure du bourgeon. Dans une seconde phase, correspondant à la formation des autopodes, les domaines d'expression s'étendent à la fois vers les extrémités distales et antérieurement de telle sorte qu'une expression postérieure se mue en expression essentiellement distale, postérieure et antérieure ([3] et figure 4B). Cette apparente rotation des domaines d'expression est donc concomitante de l'apparition des extrémités et recouvre les domaines présomptifs des mains et des pieds. D'autre part, les gènes du complexe $\mathrm{A}$ sont exprimés selon une restriction proximodistale dès le début du bourgeonnement [26, 27].

Les résultats obtenus chez le poissonzèbre tendent tous vers une même conclusion, seules donc quelques illustrations seront présentées ici. Au cours du développement précoce de la nageoire pectorale, l'expression des gènes Hoxd-10, Hoxd-11, Hoxd-12 et Hoxd-13 est pratiquement identique à la situation décrite chez les tétrapodes; alors que le gène Hoxd10 est exprimé partout dans le bourgeon, les gènes localisés plus en amont sur le complexe, tels que Hoxd-12 ou Hoxd-13, sont de plus en plus restreints à la partie postérieure du mésenchyme tout en étant complètement absents de l'ectoderme (figure 3). Cette similitude importante entre poissons et rongeurs, qui fournit donc une démonstration moléculaire des homologies entre appendices latéraux chez les vertébrés, n'est cependant pas maintenue aux stades morphogénétiques plus avancés. En effet, aucune rotation des domaines d'expression n'est observée chez les poissons, ces domaines restant confinés à la marge postérieure des nageoires, même aux stades les plus avancés. Par conséquent, aucune expression n'est détectée dans la partie antérieure des nageoires, contrairement à la situation observée dans les membres ([21] et figure 4)

Dans le cas du gène Hoxa-11, l'expression précoce dans le bourgeon de nageoire est, une fois encore, très similaire à celle décrite chez les tétrapodes, c'est-à-dire une distri- 
bution relativement homogène allant jusqu'à la partie la plus distale. Toutefois, l'expression tardive est, là encore, très différente puisque le domaine reste distal dans la nageoire, alors que les extrémités sont négatives dans les membres, le domaine étant restreint à une bande antéro-postérieure au niveau du zeugopode (figure 4). Il apparaît donc que des marqueurs de l'autopode des tétrapodes, tels que Hoxd-13, ne sont pas exprimés de façon distale chez les poissons et que, inversement, des marqueurs du zeugopode des tétrapodes, tel Hoxa-11, sont exprimés dans les parties les plus distales de la nageoire [21]. L'interprétation de ces observations dans le cadre d'un scénario possible de la transition nageoire-membre est discutée plus loin.

Chez les actinoptérygiens, les nageoires pelviennes, homologues de nos membres postérieurs, sont très différentes des nageoires pectorales. Alors que les nageoires pectorales ont un endosquelette relativement élaboré, en tout cas suffisant pour assurer une bonne articulation et le mouvement de la nageoire, les nageoires pelviennes ont un endosquelette très simple et réduit, ce qui les fait considérer comme des réitérations raccourcies des nageoires pectorales. Une prolifération très limitée du bourgeon n'entraîne la formation que de peu d'éléments osseux, très simples dans leurs formes. Contrairement aux nageoires pectorales, la plupart de ces éléments osseux ne s'articulent pas aux lépidotriches du squelette dermique, ces derniers contactant directement le basipterygium [21]. L'étude des patrons d'expression des gènes Hoxd ainsi que de sonic hedgehog (shh) au cours du développement de la nageoire pelvienne a révélé une forte corrélation entre, d'une part, la réduction extrême de l'endosquelette et un repli très précoce de la couche ectodermique et, d'autre part, une expression fugace et localisée de ces gènes [16]. En effet, les gènes Hoxd-12 et shh, par exemple, ne sont exprimés que dans quelques cellules postérieures [21].

La rencontre entre l'embryologie, la paléontologie et la génétique moléculaire du développement a permis
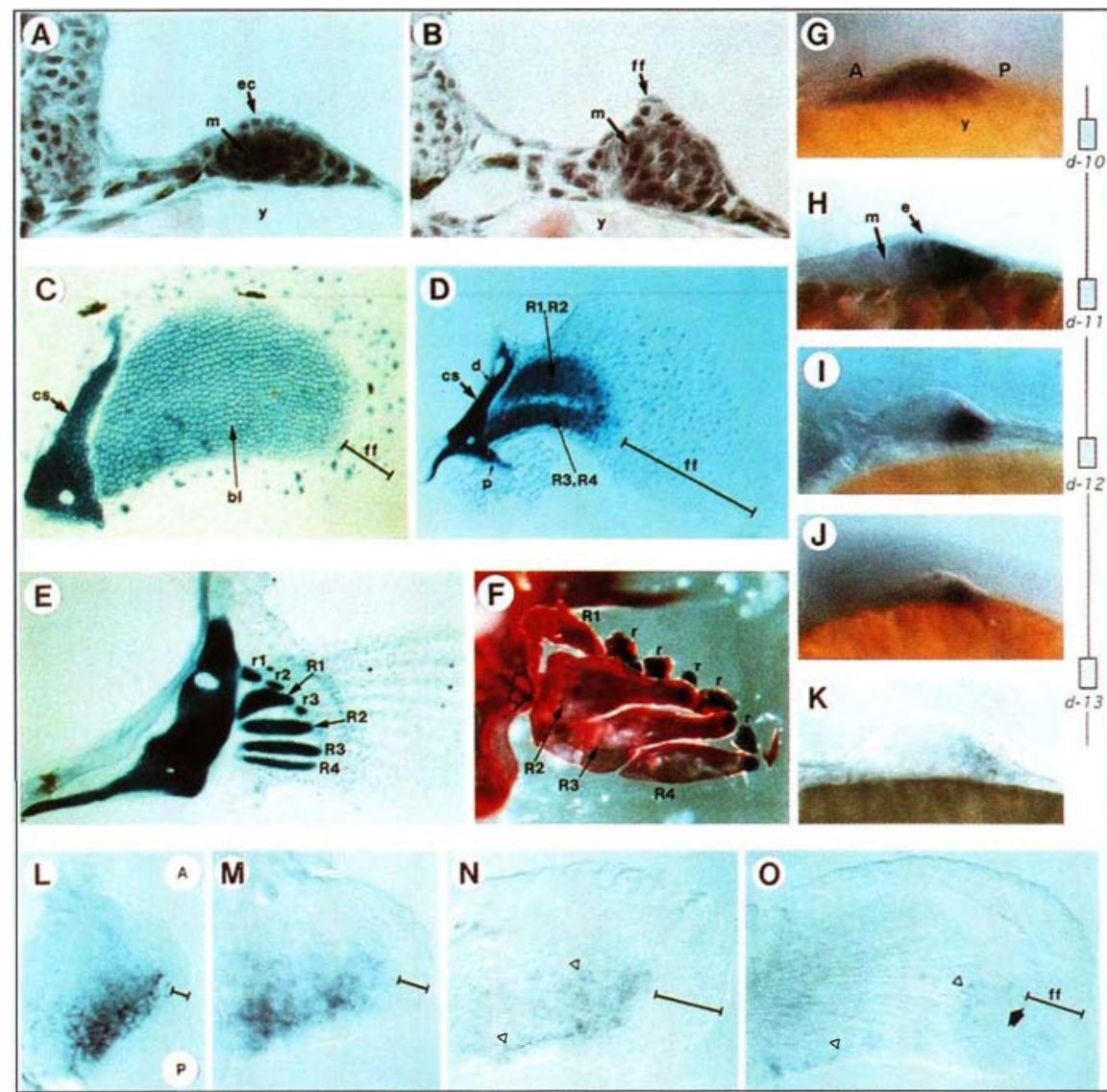

Figure 3. Développement de la nageoire pectorale de Danio et expression des gènes du complexe HoxD. A,B. Coupes histologiques de deux stades précoces du bourgeonnement, où l'on voit bien apparaître le repli ectodermique (B). $C, D, E$. Trois stades progressifs dans la chondrogenèse des éléments osseux de l'endosquelette. Une première condensation apparaît (C), puis se scinde en deux (D) puis en quatre (E). Ces quatre modèles cartilagineux représentent les quatre futurs os radiaux proximaux indiqués comme R1-R4 (F). G,H,I,J,K. Expression des gènes Hoxd-10, d-11, d-12 et d-13 dans le bourgeon précoce de la nageoire. L'expression est de plus en plus réduite à la partie postérieure, d'une façon colinéaire à la disposition des gènes sur le complexe (à droite). Ainsi, Hoxd-10 est largement distribué alors que Hoxd-13 est très restreint postérieurement. L,M,N. Expression de Hoxd-12 à des stades progressivement plus tardifs du développement de la nageoire. On observe une diminution de l'expression qui reste dans la moitié postérieure. La même observation est faite pour le gène Hoxd-13 dans une nageoire de presque trois jours (O). Seules quelques cellules sont marquées dans la partie postéro-distale. ec, ectoderme; $m$, mésoderme; ff repli ectodermique (fin fold); $c s$, cartilage coraco-scapulaire; $A$, antérieur; $P$, postérieur; $y$, vitellus. La barre noire dans $C, D, L, M, N$ et $\boldsymbol{O}$ indique l'extension du repli ectodermique. Figure reproduite avec l'autorisation de Nature, 375, 678-681, Copyright 1995 Macmillan Magazines Ltd [21].

hension de la transition nageoiremembre. Les informations accumulées aux interfaces de ces trois domaines de recherches nous suggèrent que, si les nageoires et les membres se ressemblent par certains aspects des patrons d'expression des gènes architectes, des différences essentielles sont observées. D'un côté, la persistance évolutive de la même machinerie génétique impliquée dans le développement des appendices latéraux corrobore, à un niveau moléculaire, l'homologie des nageoires des poissons actinoptérygiens et des membres des amniotes. D'un autre côté, d'importantes différences apparaissent qui illustrent la complexité croissante des morphologies, de la nageoire au membre. Ces 


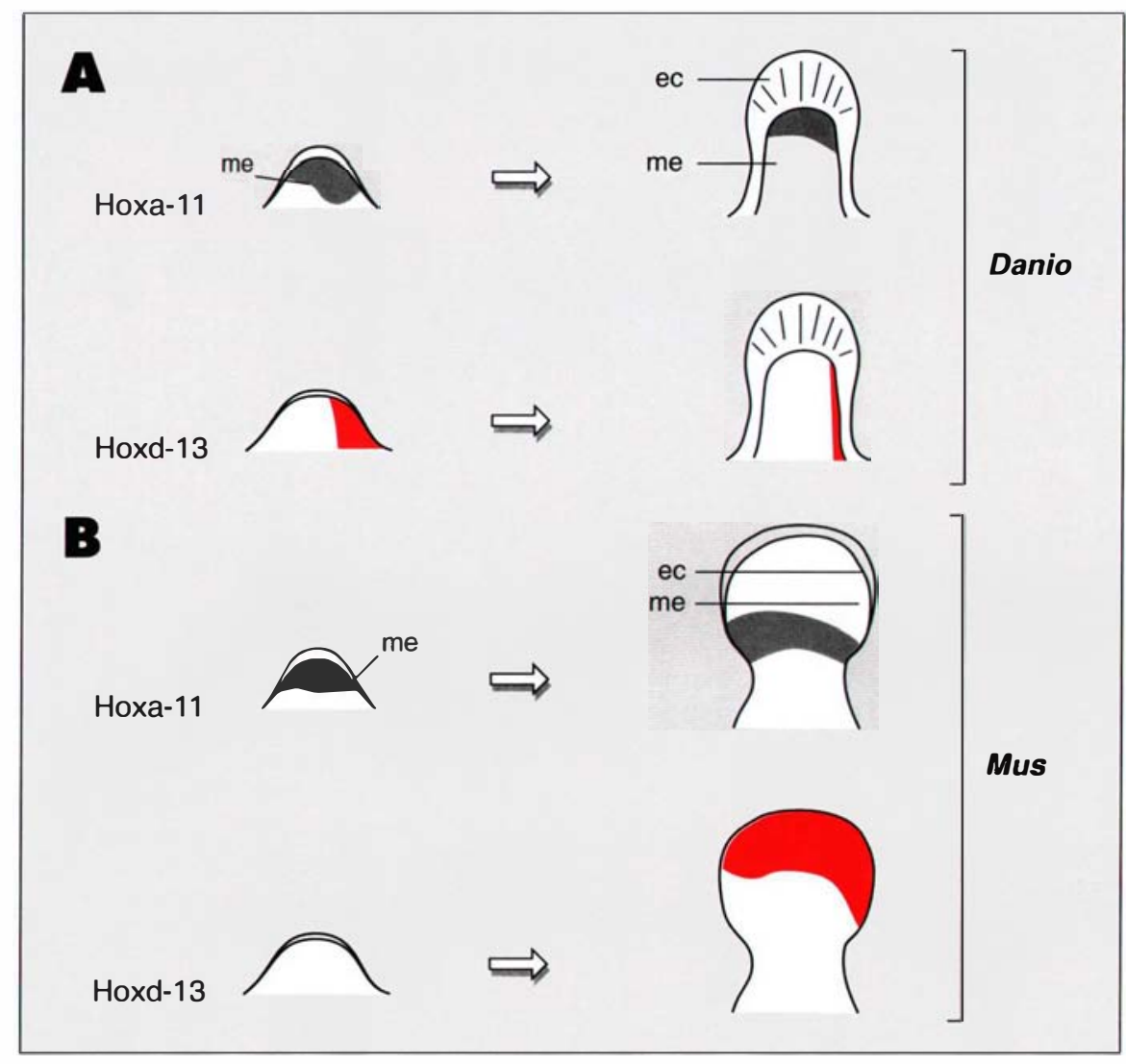

Figure 4. Schémas de l'expression des gènes Hoxd-13 et Hoxa-11 lors du développement des nageoires et des membres. Les colonnes de gauche et de droite correspondent aux phases précoces et tardives, respectivement, de la figure 2. A. Chez Danio, Hoxa-11 est exprimé dans la partie distale de la nageoire embryonnaire (à droite), alors que Hoxd-13, après une expression postérieure, garde une expression très postérieure (à droite) avant de cesser toute expression. B. Chez les tétrapodes, l'expression de Hoxa-11 est restreinte à une partie plus proximale du membre (au niveau du zygopode et du poignet), ce qui confirme le caractère néomorphe de l'autopode (une structure "moléculairement" absente de la nageoire). En dessous, l'expression du gène Hoxd-13 chez les tétrapodes devient distale, au stade tardif, et perd ainsi de sa spécificité postérieure, un changement qui n'est pas observé chez les poissons. me, mésenchyme; ec, ectoderme; l'avant est à droite, l'arrière à gauche.

différences sont naturellement d'un grand intérêt pour comprendre les bases génétiques et mécanistiques de cette transition et il apparaît d'ores et déjà que, d'un point de vue strictement moléculaire, la nageoire semble être dépourvue de la phase terminale de la morphogenèse des membres (phase tardive, figure 2). En quelque sorte, les membres peuvent être considérés comme des nageoires comportant des parties distales supplémentaires, les autopodes, ce qui n'est pas sans rappeler certains aspects de la théorie des récapitulations de Haeckel, maintenant tombée en discrédit.
Sur la base d'études morphogénétiques concernant la progression dans le temps des condensations cartilagineuses, on a suggéré que chez les amniotes, la chondrogenèse des membres se déroule en suivant un axe majeur, proximo-distal, qui est plié dans sa partie la plus distale, au niveau de l'arche digitale [14]. Cet axe serait donc homologue de l'axe métaptérygial décrit par Gegenbaur [28], allant droit de la base à l'apex, qui caractérise les nageoires des poissons vivants ou fossiles (le metapterygium fut décrit, à l'origine, dans les nageoires pectorales de requins comme le plus postérieur des os radiaux proximaux). Cette proposition novatrice impliquait que les doigts des tétrapodes, soit étaient homologues des rayons de nageoire les plus postérieurs et distaux, soit n'avaient pas de structure homologue chez les vertébrés inférieurs. Les résultats obtenus sur les gènes Hox, ainsi que nos connaissances sur leurs modes d'action [29], vont clairement à l'appui de la seconde possibilité.

Notre hypothèse de travail est donc fondée maintenant sur le scénario suivant: dans des appendices latéraux de type nageoire ancestrale, le repli ectodermique était précédé par une prolifération relativement importante et symétrique du compartiment mésenchymateux, donnant lieu à un endosquelette bien développé (probablement à plusieurs «étages»), prolongé par un squelette dermique réduit. Cet appendice était construit sur la base d'un axe métaptérygial droit. Une telle morphologie pourrait être illustrée aujourd'hui par les nageoires des poissons sarcoptérygiens tels que les dipneustes ou les coelacanthes, qui pourrait ainsi refléter cette étape de l'évolution des membres. Au cours de la phylogenèse des poissons téléostéens, le repli ectodermique se manifesta de plus en plus tôt (hétérochronie), à la suite du bourgeonnement de la nageoire, ce qui entraîna une réduction concurrente de la prolifération de la partie endosquelettique, au bénéfice d'un large squelette dermique flexible mieux adapté au milieu aquatique. Chez les tétrapodes, aucun repli n'est observé, de telle sorte que le squelette dermique est inexistant. A la place, une forte prolifération est maintenue, à un stade ultérieur, par un autre système génétique, basé principalement sur une boucle rétroactive entretenue par les produits des gènes shh (sonic hedgehog) et Fgf-4 (filbroblast growth factor 4) [30, 31] qui conduit à une surprolifération du mésenchyme postérieur et, par là même, à une croissance asymétrique de la partie distale.

Il est donc probable que les poissons ont le potentiel génétique de faire des doigts mais que la production d'un squelette dermique se fait au détriment de ceux-ci, le repli ectodermique nécessaire empêchant cette croissance postérieure terminale de se manifester. Ce scénario, relativement simple (et forcément incor- 
rect), implique que la courbure de l'axe métaptérygial des membres des tétrapodes est due à un mécanisme morphogénétique, la croissance préférentielle du mésenchyme postérieur [3, 32, 33], plutôt qu'au redressement mécanique d'une structure droite, pré existante, par exemple en réponse à de nouvelles contraintes liées à l'environnement découlant de la colonisation de substrats plus solides. Cela implique donc que les doigts sont des structures néomorphes, produites à partir de la marge postérieure des membres au cours du développement. Cette hypothèse est appuyée par l'analyse du gène Hoxa-11, dans la nageoire et dans le membre, l'expression comparative de ce gène pouvant être considérée comme une illustration moléculaire de l'absence, chez les téléostéens, de structures homologues de la partie distale des autopodes des tétrapodes. Dans ce contexte, les doigts doivent être considérés comme des spécialités de vertébrés supérieurs [34].

L'analyse des domaines d'expression des gènes $H o x$ et $s h h$ peut donc être intégrée dans des schémas d'évolution des appendices latéraux découlant de l'étude de la paléontologie et de l'analyse morphologique. Dans ce cas précis, ces études, tout en renforçant une proposition récente par des bases moléculaires, ont permis d'affiner un scénario possible de transition, de trancher en faveur d'une origine néomorphe de la digitation et de proposer une base mécanique à la courbure de l'axe des membres amniotes [20]. Cependant, ces résultats ne nous permettent pas de statuer d'une façon définitive sur l'implication fonctionnelle de ces gènes dans cette transition morphologique. De futures manipulations génétiques sur les deux systèmes (souris et poissons) devraient apporter une réponse à cette question

\section{Remerciements}

Nos remerciements vont à $\mathrm{F}$. van der Hoeven, J. Beckers, M. Coates, N. Shubin et J. Géraudie pour leurs aides et conseils ainsi qu'aux membres du laboratoire pour leurs commentaires. Ce travail est soutenu par des fonds du Fonds National Suisse de la Recherche, de l'état de Genève, du HFSPO et des fondations Claraz et Latsis.

\section{Summary}

From fins to limbs: towards a molecular approach to the evolution of vertebrate paired appendages

Over the past few years, several genes involved in the development of the tetrapod limbs have been isolated and characterized. Thus, it becomes possible to study some of the developmental mechanisms responsible for the morphogenesis of these complex structures through a novel approach. Alternatively, these genes can be used as tools to examine, at the molecular level, some aspects of the fascinating question of the fin to limb transition. Such an approach provides additional facts which illustrate both the similarities and differences in the morphologies of these homologous structures. In particular, the study of zebrafish (Danio rerio) Hox genes suggests a scenario for the fin-limb transition in which the distal parts of the autopods (the digits) are neomorphic structures produced by unequal proliferation of the posterior part of an ancestral appendix. Such an asymmetric morphogenesis could be the reason why tetrapods have their major limb axes bent anteriorly, towards their distal ends, while the homologous fin axis extends straight from proximal to distal.

\section{TIRÉS À PART}

D. Duboule.

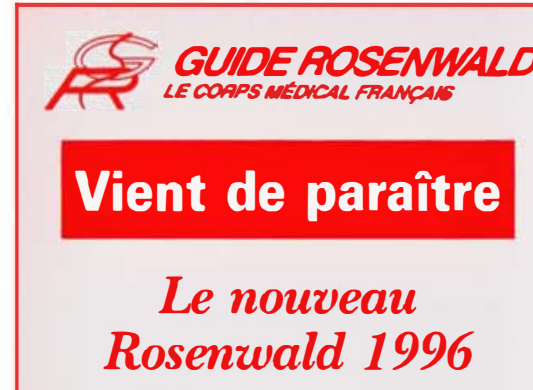

La 108 édition 1996 du GUIDE ROSENWALD vient de paraitre en 3 volumes de 5474 pages. C'est un véritable record ! Plus de la moitié des fiches des 153084 médecins et 11382 établissements recensés sont corrigées par les médecins et établissements eux-mêmes d'une édition sur l'autre. Ces informations fiables, utiles et indispensables, en permanence à portée de votre main, faciles à trouver, vous font gagner un temps précieux.

Cette année encore, le Rosenwald 1996 contient de nombreuses améliorations de contenu et de forme pour mieux satisfaire les besoins du corps médical français. Ce qui justifie la politique du Guide : satisfait ou remboursé.

- Le tome 1 (2050 pages) fournit tous les renseignements sur les 153084 médecins hospitaliers et libéraux, classés par ordre alphabétique pour la France entière.

- Le tome 2 (2030 pages), reclasse les médecins par ordre géographique et par spécialités. - Le tome 3 (1380 pages) regroupe les généralistes classés par département et les partenaires de la Santé : administrations, laboratoires et fournisseurs, hôpitaux et cliniques. Cet ouvrage complet vous aide dans votre travail quotidien. Il est irremplaçable pour constituer un carnet d'adresses, vérifier un téléphone ou un fax, rechercher un confrère ou un établissement.

Depuis des générations, les médecins utilisent le Rosenwald. Ils sont sûrs d'y retrouver rapidement tout ce qu'il faut savoir sur les médecins, les établissements de soins et les professionnels de la Santé. Chaque fiche biographique du médecin ou signalétique de l'établissement est la plus complète possible. Plusieurs classements : alphabétique, géographique, par spécialités et disciplines permettent de trouver immédiatement les renseignements précis recherchés.

Guide Resenuald, 10, nue Vinouse, 75784 Paris Cadex 16 Tél. : 44.30.81.00 - Fax : 44.30.81.11

1 volume: $465 F$, 2 volumes: $830 F$, 3 volumes : $1180 \mathrm{~F}$, le volume $460 \mathrm{~F}$ 